\title{
African Histoplasmosis in HIV-Negative Patients, Kimpese, Democratic Republic of the Congo
}

\begin{abstract}
Nestor Pakasa, Asaf Biber, Samuel Nsiangana, Désiré Imposo, Ernest Sumaili, Hypolite Muhindo, Maria J. Buitrago, Iris Barshack, Eli Schwartz
\end{abstract}

We describe a case series of histoplasmosis caused by Histoplasma capsulatum var. duboisii during July 2011January 2014 in Kimpese, Democratic Republic of the Congo. Cases were confirmed by histopathology, immunohistochemistry, and reverse transcription PCR. All patients were HIV negative. Putative sources for the pathogen were cellarbats and guano fertilizer exploitation.

$\mathrm{I}^{\mathrm{n}}$ nfection with the fungus Histoplasma capsulatum var. duboisii, also known as African histoplasmosis, was described by Dubois et al. in 1952 (1). In total, <300 cases have been reported in the literature in Africa, mostly in sporadic forms $(2,3)$.

Although the classical histoplasmosis, caused by $H$. capsulatum var. capsulatum, involves mainly the lungs, African histoplasmosis commonly involves the skin, followed by the bones. It tends to occur more frequently in patients infected with HIV. The pathogenesis of classical histoplasmosis, inhaling spores from bats' and birds' soil or guano, is well established, but the pathogenesis of African histoplasmosis remains unclear (2).

In the past, few reported cases of African histoplasmosis have been described from the Democratic Republic of the Congo; all were sporadic (3-6). We describe an unusual case series of African histoplasmosis in HIV-negative patients in Kimpese, Democratic Republic of the Congo.

\section{The Study}

All tissue samples diagnosed from histopathology as African histoplasmosis in routine biopsies at Institut Médical Evangélique Kimpese (Kimpese) during 2011-2016

Author affiliations: University of Kinshasa Hospital, Kinshasa, Democratic Republic of the Congo (N. Pakasa, E. Sumaili, H. Muhindo); Chaim Sheba Medical Center, Ramat Gan, Israel (A. Biber, I. Barshack, E. Schwartz); Institut Médical Evangélique, Kimpese, Democratic Republic of the Congo (S. Nsiangana, D. Imposo); University of Antwerp, Antwerp, Belgium (H. Muhindo); Instituto de Salud Carlos III, Madrid, Spain (M.J. Buitrago)

DOI: https://doi.org/10.3201/eid2411.180236 were included in the study. Most patients were female; median age was 20.5 years, and $42 \%$ percent were schoolage children (Table). All but 3 were residents of Kimpese. Most of the infections occurred during July 2011-October $2012(\mathrm{n}=32 ; 88.9 \%)$; case rates then sharply declined in $2013(\mathrm{n}=3 ; 8.3 \%)$, subsiding to zero after January 2014 (Table). Four patients who were available for interviews reported living in houses that were heavily infested with cellar bats; these persons frequently collected guano from cellars to fertilize gardens, although their official occupation was not agricultural.

Laboratory findings, mainly from blood and feces, were nonspecific, apart from an increased erythrocyte sedimentation rate in most patients, a feature not diagnostic per se in the tropics. HIV test results were negative for all patients.

A total of 36 consecutive routine biopsies yielded diagnoses of $H$. duboisii. Seven specimens were from skin, 7 from bones, 5 from lymph nodes, and 8 of crumbly necrotic material. The rest of the specimens were labeled tumor or tumefaction related to clinical preoperative diagnosis.

Biopsy samples were fixed in 10\% formalin and processed in the local laboratory using standard techniques of hematoxylin and eosin (HE) staining for light microscopy. Because special staining for fungi is unavailable in Kimpese, paraffin blocks were sent to Sheba Medical Center (Ramat Gan, Israel), where control HE, periodic acid Schiff (PAS), and Grocott methenamine-silver (GMS) staining were performed. Paraffin blocks were also brought to the Institut Pasteur Paris (Paris, France) for immunohistochemistry (IHC) to confirm the identity of the fungus using a noncommercial monoclonal antibody that detects both $H$. capsulatum and $H$. duboisii, distinguishable by their respective sizes ( $1-5 \mu \mathrm{m}$ vs. $7-15 \mu \mathrm{m}$ in diameter). The fungus phenotype was finally validated as H. capsulatum var. duboisii by the referral center le Centre National de Référence des Mycoses Invasives et Antifongiques in Paris. In addition, molecular analyses were performed at the Mycology Reference Laboratory, Centro Nacional de Microbiología, Instituto de Salud Carlos III (Majadahonda-Madrid, Spain), using a multiplex in-house specific real-time reverse transcription PCR (RT-PCR), as described previously (7).

In all tested samples, many intracellular or extracellular microorganisms were conspicuous on HE sections 
Table. Characteristics of patients with African histoplasmosis, Democratic Republic of the Congo, July 2011-January 2014

\begin{tabular}{lc}
\hline Characteristics & Value $^{*}$ \\
\hline Sex & $13(36.1)$ \\
$M$ & $23(63.9)$ \\
$F$ & \\
Year of diagnosis & $13(36.1)$ \\
2011 & $19(52.8)$ \\
2012 & $3(8.3)$ \\
2013 & $1(2.8)$ \\
2014 & $20.5(10.5-39.0)$ \\
\hline Age, y, median (interquartile range) & $15(41.7)$ \\
$3-16$ & $15(41.7)$ \\
$17-49$ & $6(16.6)$ \\
$\geq 50$ & \\
\hline *Values are no. (\%) except as indicated.
\end{tabular}

(Figure, panel A). In most cases, organisms were seen in the cytoplasm of multiple multinucleated Langhans-type giant cells, often dividing by explosive budding (Figure, panel A) and frequently demonstrating explosive giant asteroid bodies (Figure, panel B), at times undergoing degeneration. The fungus was easily identified on PAS (Figure, panel C) and GMS (Figure, panel D).

Twelve samples from different patients underwent further IHC staining, which revealed a membranous staining of large 7-15- $\mu \mathrm{m}$ yeasts. RT-PCR assays performed in paraffin-embedded tissue samples from 3 patients were all positive for $H$. capsulatum. The technique was unable to differentiate between $H$. capsulatum var. capsulatum and var. duboisii, because the specific probe was designed to detect both. The average fungal burden detected was 7.6 fg DNA $/ \mu \mathrm{L}$.

\section{Conclusions}

This histology-based study identified a novel focus of H. duboisii in the Democratic Republic of the Congo in the city of Kimpese and its vicinity. Over a period of $\approx 2$ years, 36 cases were routinely detected in a single pathology laboratory in this area; all patients were HIV negative.

The putative source of infection appears to be cellar bats, Chaerephon pumilus, and guano fertilizer overexploitation. African histoplasmosis has previously been reported to be isolated from the intestinal contents of bats belonging to the species Nycteris hispida and Tadarida pumila from a cave in rural Nigeria and from soil mixed with bat guano (8).

The subsiding of new cases could be related to the incidental cessation of the use of bat guano along with the reintroduction of chemical fertilizers. This change was unrelated to any knowledge about the infection risk of guano and may be reversed when the guano accumulates again to sizable amounts.

This case series featured a high incidence of infection in women and girls and in young children, including toddlers and school-age children. Six patients were 3-7 years of age, an age when children are more tied to their mothers, sit on the ground, and may be in contact with contaminated soil. Although HIV tests were negative, other innate or acquired immunosuppression was not ruled out.

During the first year of our case series, more cases were initially labeled as lymphoproliferative disorders

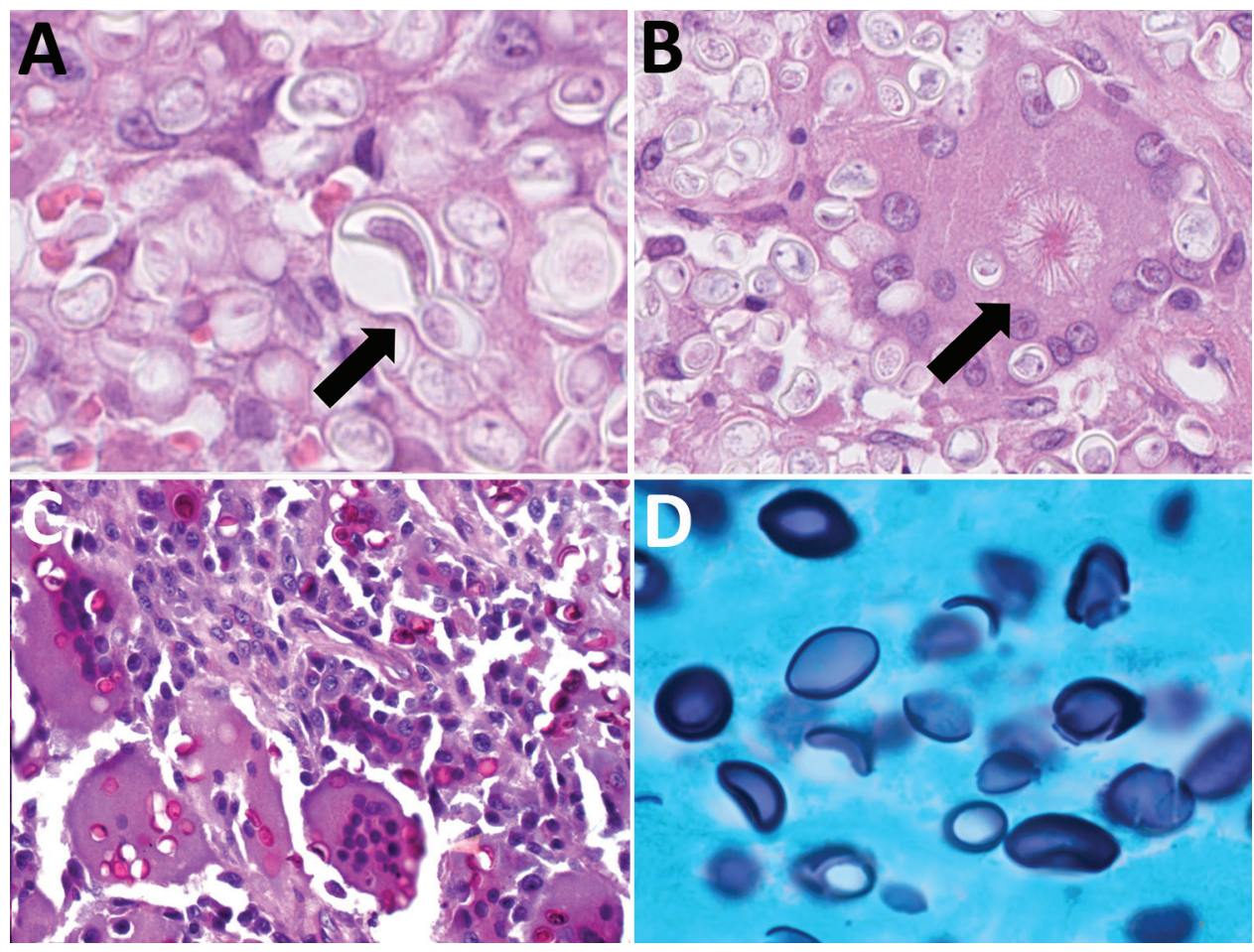

Figure. Pathologic findings from patients infected with African histoplasmosis, Democratic Republic of the Congo, July 2011-January 2014. A) Yeast explosive budding (arrow) (hematoxylin and eosin [HE] staining; original magnification $\times 160$ ); B) asteroid bodies (arrow) (HE staining; original magnification $\times 160$ ); C) yeasts in Langhans cells (periodic acid Schiff staining; original magnification $\times 160$ ); D) lemonshaped appearance (Grocott methenamine-silver staining; original magnification $\times 80$ ) 
or extrapulmonary tuberculosis, whereas in subsequent years, cases were initially suspected to be histoplasmosis infections. Therefore, it is reasonable to assume that, in endemic areas, where histopathological diagnosis or advanced microbiology labs are not widely accessible, the disease may be underdiagnosed; therefore, the prevalence of African histoplasmosis may be underestimated.

The diagnosis of African histoplasmosis is made mainly by histopathology, which shows granulomatous inflammation and giant cells containing numerous large yeast cells $10-15 \mu \mathrm{m}$ in diameter, which are thick-walled and divided by narrow budding. The fungus is easily visible by GMS, Gridley, or PAS staining (4). IHC staining, which was performed on 12 specimens, is not specific for African histoplasmosis but to $H$. capsulatum; therefore, it may play a role in more accurate and faster diagnosis and enable exclusion of other fungal infections.

In our series, 3 samples were tested by RT-PCR and results were positive in all 3 for $H$. capsulatum diagnosis. Newer PCR protocols have been developed and may provide rapid and species-specific diagnosis (9).

Our histopathologic observation has some limitations. IHC and the RT-PCR primers that were used are unable to differentiate between $H$. capsulatum var. capsulatum and var. duboisii, yet they confirmed the histoplasmosis diagnosis, and IHC together with the large size of the yeast make it specific for H. duboisii. Soil and the bat guano from the infected areas have not yet been tested for $H$. capsulatum var. duboisii. Among the strengths of this study is that this unusual case series is unique in its extent and clustering of cases. In addition, the available data add novel epidemiologic and diagnostic information to the current knowledge about this neglected disease.

Further investigation should be conducted to understand the reservoir of the pathogen, the types of daily activities that might pose risk factors for acquiring the disease, and the mode of transmission and progression of the disease. Because diagnosis, especially in these rural areas, is challenging, seroepidemiologic surveys are needed to establish the extent of this infection.

\section{About the Author}

Dr. Pakasa is a professor of pathology and histology at the University of Kinshasa, Democratic Republic of the Congo, and head of the division of nephropathology and uropathology in the department of pathology. His research interests include tropical infectious nephropathology, including podocytopathies, HIV and APOL-1 associated kidney diseases, and the pathology of neglected tropical diseases, including schistosomiasis and African histoplasmosis.

\section{References}

1. Dubois A, Janssens PG, Brutsaert P, Vanbreuseghem R. A case of African histoplasmosis; with a mycological note on Histoplasma duboisii n. sp. [in French]. Ann Soc Belg Med Trop. 1952;32:569-84.

2. Gugnani HC. Histoplasmosis in Africa: a review. Indian J Chest Dis Allied Sci. 2000;42:271-7.

3. Loulergue P, Bastides F, Baudouin V, Chandenier J, Mariani-Kurkdjian P, Dupont B, et al. Literature review and case histories of Histoplasma capsulatum var. duboisii infections in HIV-infected patients. Emerg Infect Dis. 2007;13:1647-52. http://dx.doi.org/10.3201/eid1311.070665

4. Tsiodras S, Drogari-Apiranthitou M, Pilichos K, Leventakos K, Kelesidis T, Buitrago MJ, et al. An unusual cutaneous tumor:African histoplasmosis following mudbaths: case report and review. Am J Trop Med Hyg. 2012;86:261-3. http://dx.doi.org/ 10.4269/ajtmh.2012.11-0557

5. Therby A, Polotzanu O, Khau D, Monnier S, Greder Belan A, Eloy O. Aspergillus galactomannan assay for the management of histoplasmosis due to Histoplasma capsulatum var. duboisii in HIV-infected patients: education from a clinical case [in French]. J Mycol Med. 2014;24:166-70. http://dx.doi.org/10.1016/ j.mycmed.2014.01.002

6. Chandenier J, Goma D, Moyen G, Samba-Lefèbvre MC, Nzingoula S, Obengui, et al. African histoplasmosis due to Histoplasma capsulatum var. duboisii: relationship with AIDS in recent Congolese cases [in French]. Sante. 1995;5:227-34.

7. Gago S, Esteban C, Valero C, Zaragoza O, Puig de la Bellacasa J, Buitrago MJ. A multiplex real-time PCR assay for identification of Pneumocystis jirovecii, Histoplasma capsulatum, and Cryptococcus neoformans/Cryptococcus gattii in samples from AIDS patients with opportunistic pneumonia. J Clin Microbiol. 2014;52:1168-76. http://dx.doi.org/10.1128/JCM.02895-13

8. Gugnani HC, Muotoe-Okafor FA, Kaufman L, Dupont B. A natural focus of Histoplasma capsulatum var. duboisii is a bat cave. Mycopathologia. 1994;127:151-7. http://dx.doi.org/10.1007/ BF01102915

9. Pellaton C, Cavassini M, Jaton-Ogay K, Carron PN, Christen-Zaech S, Calandra T, et al. Histoplasma capsulatum var. duboisii infection in a patient with AIDS: rapid diagnosis using polymerase chain reaction-sequencing. Diagn Microbiol Infect Dis. 2009;64:85-9. http://dx.doi.org/10.1016/j.diagmicrobio.2009.01.001

Address for correspondence: Asaf Biber, The Chaim Sheba Medical Center, The Center for Geographic Medicine and Tropical Diseases, Tel Hashomer-Ramat-Gan 52621, Israel; email: asafbib@gmail.com 